\title{
Desafío de los nuevos escenarios en un ambiente educativo en el Modelo Proactivo
}

\section{Challenge of the new scenarios in an educational environment in the Proactive Model}

\author{
Carlos Enrique Santos Loor \\ 1 \\ Universidad Técnica de Manabí. \\ Ecuador \\ https://orcid.org/0000-0001-7187-6163
}

Alba Verónica Barreto Pincay

veronicabarreto2028@gmail.com

Escuela de Educación Básica José Antonio de Vallejo

Ecuador

https://orcid.org/0000-0001-8682-5406

Flor María Bailón López

florbailon_13@hotmail.com

Unidad Educativa Norma Guerrero de Castillo

Ecuador

https://orcid.org/0000-0002-9424-0624

María Monserrate Bravo Guerrero

mabrague@hotmail.com

Unidad Educativa Rocafuerte

Ecuador

https://orcid.org/0000-0001-6163-5737

\begin{abstract}
RESUMEN
La investigación tuvo como objetivo caracterizar los desafíos de los nuevos escenarios en un ambiente educativo en el modelo proactivo en la unidad educativa del milenio "Guadalupe Larriva" de la ciudad de Jaramijo de la provincia de Manabí, mediante una metodología descriptiva. Se puede apreciar que es necesario, profundizar las fortalezas que tienen los nuevos escenarios en un ambiente proactivo para un desarrollo del proceso en los aprendizajes y así poder lograr los estándares de calidad, en que la sociedad requiere en sus educandos. Cuando los docentes trabajan desde nuevos horizontes epistémicos y pedagógicos, se puede conciliar un mejor aprendizaje basado en lo sistémico como mediación en alcanzar una posición crítica - reflexiva por parte de los estudiantes, siendo este un logro importante para lograr una educación de calidad.
\end{abstract}


Descriptores: Aprendizaje activo; Investigación centrada en un problema; Educación básica; Educación permanente; Psicología de la educación.

\section{SUMARY}

The research aimed to characterize the challenges of the new scenarios in an educational environment in the proactive model in the millennium education unit "Guadalupe Larriva" of the city of Jaramijo in the province of Manabí, using a descriptive methodology. It can be seen that it is necessary to deepen the strengths of the new scenarios in a proactive environment for the development of the learning process and thus be able to achieve quality standards, which society requires in its students. When teachers work from new epistemic and pedagogical horizons, better systemic-based learning can be reconciled as mediation in reaching a critical reflective position on the part of students, this being an important achievement to achieve quality education.

Descriptors: Activity learning; Mission oriented research; Basic education; Lifelong education. Educational psychology.

\section{INTRODUCCIÓN}

Los escenarios y el ambiente donde se desarrollan los aprendizajes han sido unos de los factores críticos para el sistema educativo de acuerdo a los resultados obtenidos en las diversas instituciones educativas en el Ecuador, con mayor frecuencia en la zona rural. En la actualidad el sistema ecuatoriano ha considerado como un desafío de mejorar los diversos escenarios educativos, creando un ambiente proactivo dotando de todas las herramientas necesarias de acuerdo a los avances tecnológicos para hacer un aprendizaje exquisito de mucha efectividad.

Las Unidades educativas del milenio y siglo XXI deben atender a la exigencia de los estudiantes de los diferentes niveles del sistema educativo y en la formación del docente comprometido en diseñar espacios estructurados, organizados para propiciar la participación activa en el desarrollo de todas las actividades académicas de los niños y niñas, favoreciendo su interacción e interactividad en los procesos de los aprendizajes, a la vez responder a las nuevas necesidades con recursos didácticos, tecnológicos para lograr un aprendizaje significativo y funcional, logrando una educación de calidad y calidez. 
La globalización está transformando profundamente la vida de las personas, descubriendo una sociedad más interactiva, dinámica y participativa de acuerdo a los avances científicos, tecnológicos y la internacionalización de las relaciones comérciales de los pueblos. En la última década el Ecuador ha realizado transformaciones trascendentes a partir del desarrollo del proyecto de Plan Decenal del 2006-2016 con el objetivo de asegurar la incorporación, actualización, utilización y apropiación crítica y reflexiva de las TIC en el proceso formativo, por parte de todos los actores y de los diferentes niveles del sistema educativo, que además favorezca la divulgación del conocimiento, teniendo en cuenta la superación de las desigualdades económicas, regionales, étnicas, de género y de las condiciones de vulnerabilidad.

Fortalecer en las instituciones y centros educativos rurales y urbanos, la infraestructura tecnológica y la conectividad con calidad y equidad, desarrollando modelos e innovaciones educativas y pedagógicas que promueven el aprendizaje activo, la interacción de los actores educativos y la participación de los estudiantes. (MEC, 2006-2015).

Con el compromiso y la continuidad del proyecto del Plan Decenal 2016-2026 con la participación de los docentes en nueva jornada de mesas de trabajo conjuntamente con la política del estado ecuatoriano basados en los resultados de las evaluaciones del plan decenal 2006-2016, para la ejecución de este proceso busca transformar las instituciones educativas en una comunidad de aprendizaje que fortalezca la identidad de los estudiantes, desarrolle integralmente sus capacidades y su compromiso social. (MEC, 2016-2026).

El desarrollo de este proyecto de transformar las instituciones educativas conjuntamente con todos los actores del ámbito educativo, con una propuesta participativa, que procura satisfacer las necesidades de la formación del estudiante que debe contribuir a transformar la sociedad, con identidad, criterio reflexivo, crítico, solidario y democrático. El perfeccionamiento del proceso de aprendizaje se constituye entonces un desafío fundamental para las instituciones educativas creando nuevos escenarios en un ambiente educativo en el modelo proactivo. 
Los programas de formación que se desarrollen deberán ser procesos continuos de aprendizaje activo y reflexivo, atendiendo a la formación cognitiva como afectiva, y motivacionales, de la personalidad del sujeto en formación, que propicien una representación del rol, fundada no solo en conocimientos sino, también en actitudes, vivencias, motivaciones y valores que le permitan una actuación profesional ética y responsable.

Se hace necesario, entonces, proporcionar investigaciones pedagógicas relacionadas a los nuevos escenarios en un ambiente educativo para la formación de los niños, niñas y jóvenes, para enfrentar los retos de la sociedad contemporánea, atinentes al perfeccionamiento del desempeño profesional en su práctica docente. La propuesta del Ministerio de Educación está enfocadas a mejorar los resultados de aprendizaje medido a través de un sistema integral de evaluación de la calidad, es decir, la evaluación como parte integral del proceso de enseñanza-aprendizaje. Para el efecto, se ha establecido: (1) actualizar periódicamente, de forma relevante y pertinente, el currículo y los estándares educativos sobre la base de los resultados del aprendizaje; y (2) consolidar un sistema integral de evaluación que considere estándares nacionales e internacionales.

De esta forma, los estudiantes deben cumplir con estándares educativos óptimos y las instituciones escolares deberán desarrollar estrategias para facilitar el logro de estas metas de aprendizaje. De La misma manera la formación docente en los nuevos diseños curriculares, en su mejora de su remuneración e incrementar o mejorar sus infraestructuras físicas de las instituciones educativas tanto en las zonas urbanas y rurales.

En la dimensión académica se admite el desarrollo de las competencias en los nuevos escenarios en un ambiente en el aula, donde los niños, niñas y jóvenes adquiere los conocimientos, habilidades, valores, con la aplicación de la tecnología generando la participación activa en el desarrollo de todas las actividades pedagógicas de los estudiantes favoreciendo su interacción e interactividad en los procesos de los aprendizajes, haya el respeto y que compartan los materiales, espacios de investigación entre sus compañeros. 
La dimensión pedagógica permitirá que los docentes propicien escenarios en un ambiente proactivo en función del objeto de estudio y a las características de los estudiantes para desarrollar las competencias donde puedan solucionar los problemas, e incluso aquellos no determinados.

La dimensión investigativa es que los docentes crean en los nuevos escenarios en un ambiente proactivo, con métodos y técnicas propias en trabajos colaborativos destacando la interactividad entre estudiantes y docente, logrando una motivación intrínseca e extrínseca para cumplir con los objetivos de los aprendizajes; es una de las competencias más generales que deben caracterizar el modo de actuación de un docente.

En estos tres espacios, hace que el futuro profesional en la docencia, fomente la investigación y la creatividad en los nuevos desafíos en los escenarios en un ambiente en el modelo proactivo para lograr los estándares de calidad que el Ministerio de educación busca en las instituciones educativas. Las Unidades Educativas del siglo XXI y las del Milenio buscan satisfacer las necesidades de los estudiantes y de los docentes con espacios estructurados y organizados para cumplir diversas actividades, de tal manera que, facilite un ambiente en que se puedan cumplir los objetivos de los aprendizajes. Así tenemos la Unidad Educativa del Milenio "Guadalupe Larriva" del Cantón Jaramijo de la provincia de Manabí, cuenta con diversas áreas de recreación, laboratorios, biblioteca y salones de clases.

Las prácticas pedagógicas deben ser una de las necesidades de los docentes en fomentar espacios estructurados y organizados para propiciar la participación activa en el desarrollo de todas las actividades académicas de los niños y niñas, favoreciendo su interacción e interactividad en los procesos de los aprendizajes colaborativo, utilizando una metodología activa, técnicas e instrumentos que permitan detectar y resolver problemas propios de la práctica profesional del aula, lo cual permite contar con un enfoque centrado en el estudiante tal como lo proyectan Peche Cruz \& Giraldo Supo (2019), quienes señalan que desde esta vertiente el estudiante se constituye en un ser activo en los ambientes de aprendizaje. 
El trabajo tiene como objetivo caracterizar los desafíos de los nuevos escenarios en un ambiente educativo en el modelo proactivo en la unidad educativa del milenio "Guadalupe Larriva" de la ciudad de Jaramijo de la provincia de Manabí.

\section{METODOLOGÍA DE LA INVESTIGACIÓN}

La investigación fue de tipo descriptiva (Hernández Sampieri, Fernández Collado \& Baptista 2014), lo cual permitió caracterizar los desafíos de los nuevos escenarios en un ambiente educativo en el modelo proactivo en la unidad educativa del milenio "Guadalupe Larriva" de la ciudad de Jaramijo de la provincia de Manabí, aplicándose una encuesta a la muestra poblacional conformada por 31 docentes.

\section{RESULTADOS}

A continuación se presentan los resultados:

¿Cómo considera usted los nuevos escenarios en un ambiente de aprendizaje de los niños y niñas y jóvenes en la institución?

\section{Grafico 1}

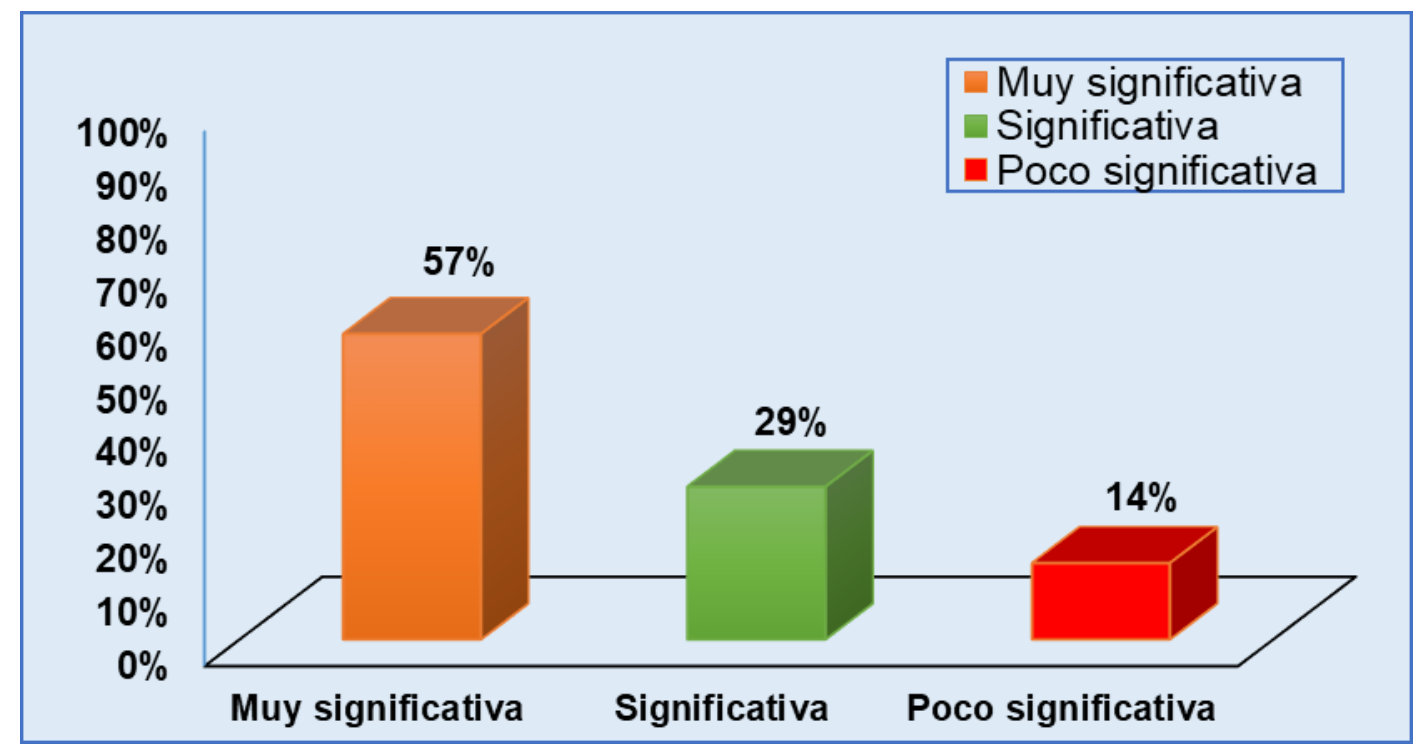

Fuente: Encuesta aplicada a los docentes (2019) 
El $57 \%$ de los encuestados considera de forma muy significativa el empleo de nuevos escenarios pedagógicos en los ambientes de aprendizaje, mientras que el $29 \%$ lo hace de forma significativa y un $14 \%$ en el renglón poco significativa, esto implica que aún existen docentes que no brindan la debida importancia de tener una apertura a nuevos desafíos con la finalidad de promover una educación proactiva en beneficio de estimular un mejor aprendizaje en los estudiantes, esto se compagina con lo planteado por Maya, Aldana Zavala \& Isea Argüelles (2019), quienes destacan la importancia de promover una educación de calidad, basada en la promoción de nuevos estilos de aprendizaje y liderazgos, para lo cual, los directivos, docentes, de la institución educativa juegan un factor primordial en tal fin.

¿Qué nivel de aprendizaje es alcanzado por los estudiantes con la utilización de los nuevos escenarios en un ambiente educativo proactivo?

\section{Grafico 2}

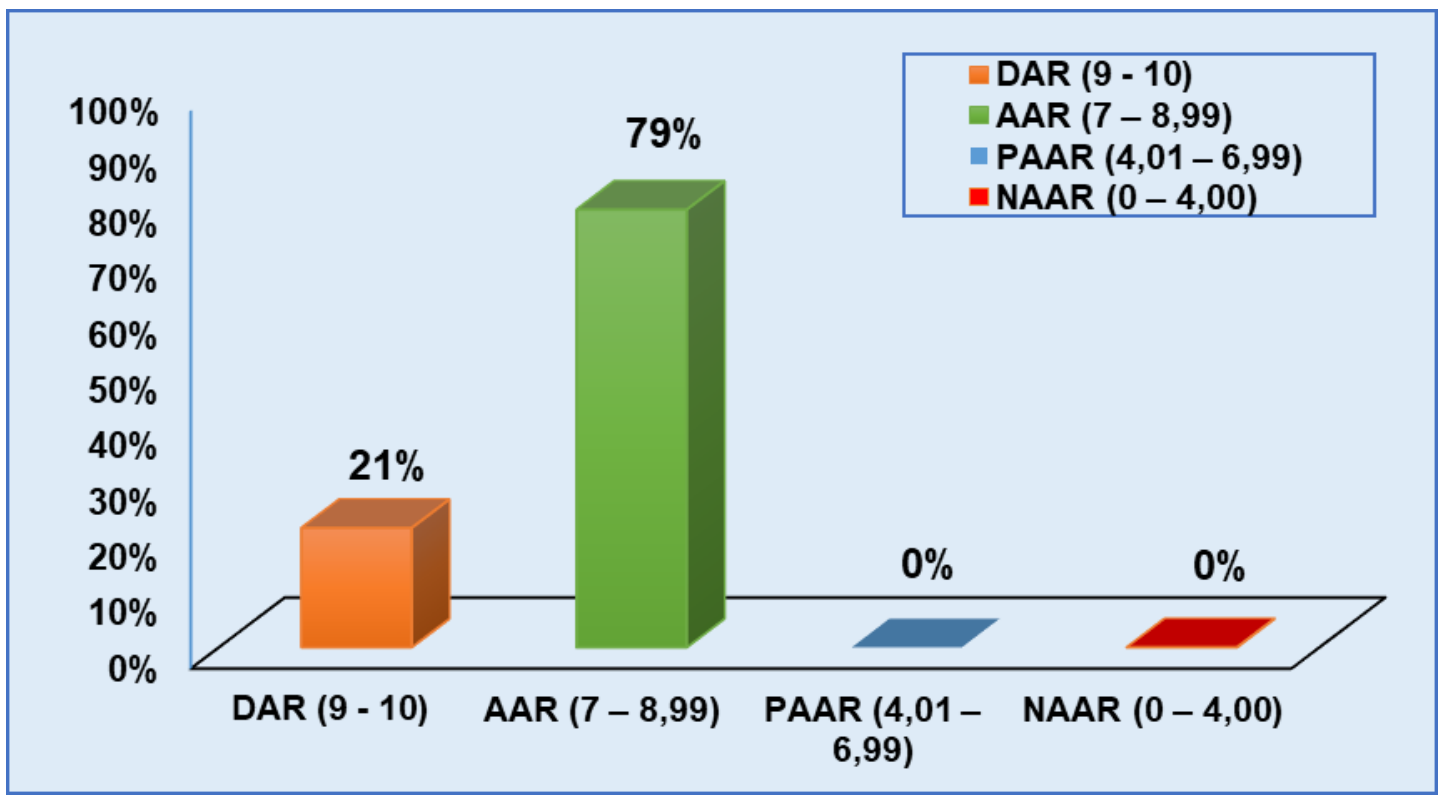

Fuente: Encuesta aplicada a los docentes (2019)

Las respuestas se focalizan en el rango comprendido entre 9-10, lo cual representa un $21 \%$ de la muestra poblacional, mientras que en el rango 7-8,99 es representando por el $79 \%$, esto indica que los estudiantes han podido superar sus 
calificaciones al encontrarse en aprobados, siendo significativo para el establecimiento de aprendizajes valorados por parte de los estudiantes. Esto se relaciona por lo planteado por Morgado, Aldana Zavala \& Isea Argüelles (2019), quienes plantean que es necesario en las instituciones educativas planificar esfuerzos que permitan trabajar en base a una gerencia transformacional, siendo el docente un gerente de los ambientes de aprendizaje, con la finalidad de promover en los estudiantes transformaciones para adoptar cambios favorables que le permitan crecer constantemente como personas integrales en capacidad de asumir los retos sociales inherentes a los continuos cambios producto del dinamismo global que impulsa a las sociedad a estar en un proceso constante de renovación.

¿En que fortalece el sistema educativo en el empleo de los nuevos escenarios en un ambiente proactivo?

\section{Grafico 3}

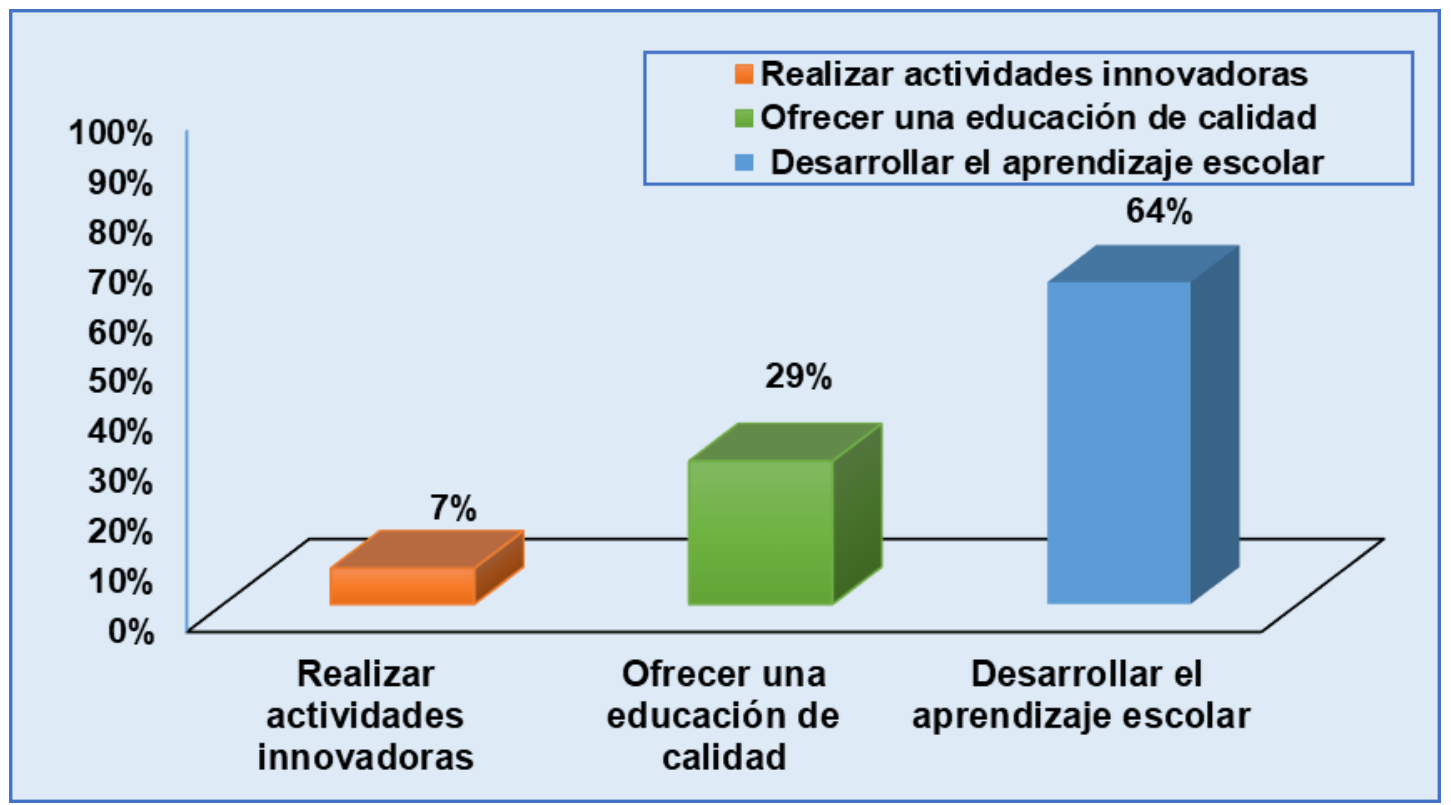

Fuente: Encuesta aplicada a los docentes (2019)

Un 7\% plantea que realizar actividades innovadoras fortalece el sistema educativo, un $29 \%$ considera que se ofrece una educación de calidad, mientras que el $54 \%$ 
plantea que brinda mayor aporte el desarrollar el aprendizaje escolar. Esto implica estar en concordancia con lo planteado por Aldana, Salón \& Guzmán (2019), al expresar que cuando los docentes trabajan desde nuevos horizontes epistémicos y pedagógicos, se puede conciliar un mejor aprendizaje basado en lo sistémico como mediación en alcanzar una posición crítica - reflexiva por parte de los estudiantes, siendo este un logro importante para lograr una educación de calidad.

\section{CONCLUSIONES}

A partir de los resultados planteados, se puede apreciar que es necesario, profundizar las fortalezas que tienen los nuevos escenarios en un ambiente proactivo para un desarrollo del proceso en los aprendizajes y así poder lograr los estándares de calidad, en que la sociedad requiere en sus educandos.

Considerando la naturaleza de la teoría y la practica educativa como del conocimiento, lo verifica, así como el docente es el responsable en la transformación de los procesos en los aprendizajes, atendiendo a las necesidades de los estudiantes para propiciar espacios con los cuales se les da objeto de estudios para fomentar discusiones entre estudiantes en el momento de explorar definiciones que ameritan consensuar situaciones problemáticas que desea resolver, se busca la combinación e interacción de situaciones e interacciones sociales que puedan fortalecer un aprendizaje personal, grupal y efectivo. Los nuevos escenarios educativos son el "entorno, físico o virtual, donde se genera las necesarias interacciones entre diferentes actores y agentes educativos, logrando, de este modo, el aprendizaje" (Gallardo, 2017).

Por consiguiente, "Se considera espacio físico al lugar en el que se sitúan los objetos y/o el marco físico en el que se suceden las actividades que llevan a cabo los individuos; el espacio físico es un concepto afín al espacio objetivo; el espacio externo, es todo el lugar que lo rodea" (Córdova, 2011). Por lo que se puede manifestar que los escenarios educativos son espacios estructurados y organizados con fines educativos, de tal manera que, facilite el acceso del desarrollo de todas las actividades de los niños, niñas y jóvenes, favoreciendo su interacción en los procesos de los aprendizajes. Mientras que los ambientes educativos es una 
participación activa en el desarrollo de todas las actividades de los niños y niñas, favoreciendo su interacción e interactividad en los procesos de los aprendizajes.

De esta forma, se puede mencionar que, gracias al aporte pedagógico de los modelos de enseñanza actual, se están creando nuevos escenarios educativos ideales para garantizar el desarrollo del aprendizaje de manera significativa y funcional; es importante que, a los estudiantes, se les brinde espacios donde puedan interactuar y adquirir habilidades/destrezas. "Los nuevos escenarios educativos, como cualquier otro ámbito de la sociedad actual, se enfrenta a los cambios sociales y tecnológicos que se producen a una velocidad vertiginosa" (Blasco \& Pérez, 2012). La implementación de nuevos escenarios educativos adecuados para la educación siempre requiere: "comodidad, facilidad para estudiar solo y en grupos, comunicación cordial, acogida, limpieza, orden, pulcritud y silencio; el sentirse a gusto en este ambiente lleva a sentirse seguro, aceptado, val valorado, querido por quienes conviven en él, esto refuerza sentimientos de seguridad, dignidad y solidaridad" (Merma, 2012).

Las instituciones educativas deben contar con nuevos entornos educativos seguros, donde los estudiantes y docentes puedan ejecutar actividades formativas y por ende alcanzar el máximo nivel de aprendizaje requerido. Con la aplicación de la pedagogía en la educación, se ha logrado "convertir los nuevos escenarios educativos en cualquier espacio útil para el propósito formativo y aprovechando aquel agente educador que tenga algo que aportarle a la formación" (Ramírez, 2013).

Los nuevos escenarios educativos tecnológicos, "se construyen con una medicación pedagógica a partir de las interacciones y las intervenciones entre docentes y estudiantes, sus relaciones establecidas con los objetivos tecnológicos, así como los proceso de aprendizaje constituidos por la integración de los aspectos señalados" (García, 2014).

Las tecnologías de la información propician el surgimiento de nuevos espacios para aprender de manera interactiva a través de la interacción entre los miembros de la comunidad educativa. Por ello es importante, "incluir herramientas tecnológicas en 
los escenarios educativos (computadores, vídeo, tableros digitales, tabletas, software educativos, entre otros)" (Lara, 2015).

En definitiva, es relevante mencionar que la tecnología aporta en la generación de los nuevos escenarios educativos, que debe estar al alcance de todos los actores del sistema educativo, para fortalecer la construcción de aprendizajes activos. El aprendizaje es activo porque: "una exigencia básica para la estructuración del proceso de enseñanza-aprendizaje es precisamente la búsqueda activa del conocimiento por parte del estudiante, teniendo en cuenta las acciones didácticas a realizar por éste" (Ortiz, 2010).

El aprendizaje es de tipo cooperativo en el "entorno en que los estudiantes trabajan en grupos de habilidades mixtas y se les recompensa con base en el éxito del grupo" (Ortiz, 2010). Este aprendizaje nace con el constructivismo social y se basa en la adquisición de conocimientos a través de la ejecución de actividades en las que todos los estudiantes aprenden conjuntamente. El aprendizaje creativo cuando "el estudiante se apropia de los contenidos, habilidades y normas de comportamiento que les permiten la aplicación creativa a nuevas situaciones de aprendizaje" (Ortiz, 2010).

Los estudiantes denotan este tipo de conocimiento cuando ejecutan acciones educativas con ideas propias; es así que van aprendiendo a un ritmo libre, voluntario, dinámico, guiado siempre por el docente durante las jornadas educativa. Desde esta perspectiva, la presente trabajo cuenta de los análisis valorativos realizados en la investigación desarrollo por el autor principal, que, teniendo como objetivo aportar a la comprensión de una dimensión específica de la actividad docente estudiante, reflexión colectiva que se deben desarrollar con los profesores activos y en formación, sobre los desafíos de los nuevos escenarios en un ambiente proactivo para potenciar una manera particular a los nuevos profesores, y resolver los problemas subyacentes a la práctica pedagógica y su posibilidad de alcanzar los estándares de calidad.

La sistematización de la experiencia en la actividad desarrollada por los docentes, posibilita percibir los estilos de práctica y acceder a una descripción objetivada de ella. Cuando se reflexiona sobre la práctica de los nuevos escenarios en un 
ambiente proactivo, contrastándola con fuentes teóricas o visiones provenientes de los de los docentes con la finalidad problematizar los nuevos estilos práctica, este proceso permite fortalecer el conocimiento individual de los actores en los aprendizajes hacia la construcción de un tipo de saber consensual. Desde allí emerge el Saber del desafío de los nuevos escenarios en un ambiente proactivo.

La pedagogía actual, hace, que cada día el docente debe ser más exigente a las necesidades del estudiante, para cumplir con los requerimientos de los estándares de calidad en el sistema educativo, ya que estos no están suficientemente fortalecidos en diseñar escenarios en un ambiente proactivo, existiendo también limitaciones por quienes tienen la responsabilidad de brindar estos escenarios a todas las instituciones educativas.

Para poder indicar la innovación de la didáctica pedagógicas, los docentes deben ser orientados o actualizados sobre los diseños de los nuevos escenarios en un ambiente proactivo, en las diversas áreas o disciplinas y el compromiso de partes quienes administran las instituciones educativas.

Las instituciones educativas deben de disponer con espacios físicos adecuados para que los docentes puedan incentivar a los niños a realizar actividades a través de las cuales puedan aprender diversos contenidos temáticos relacionados con las asignaturas contempladas en la malla curricular; los mismos que serán evaluados por los docentes para obtener resultado del nivel de aprendizaje alcanzado. Como lo menciona (Navarro, 2014), de la gestión educativa como "el conjunto de acciones, realizada por los actores escolares, que están orientadas hacia el cumplimiento de la tarea fundamental de la escuela, la generación de condiciones, ambientes y procesos necesarios para que los estudiantes aprendan".

\section{REFERENCIAS CONSULTADAS}

1. Aldana, J., Salón, M., \& Guzmán, N. (2019). Liderazgo sistémico en las competencias gerenciales docentes universitarias. CIENCIAMATRIA, 5(8), 
2. ARRANZ, M., \& GARCIA, C. (2011). Didáctica de la Educación Infantil. España: Paraninfo.

3. BIXIO, Cecilia. - Nuevos-Escenarios-Educativos. Año 2010.

4. BLASCO, J., \& PEREZ, J. (2012). Metodología de investigación en las ciencias de la actividad física y el deporte. España: ECU

5. CAUSIL GONZÁLEZ, Gustavo Adolfo. - Ambiente Educativo. Año 2013.

6. CORDIVA, D. (2011). Desarrollo cognitivo, sensorial, motor y psicomotor en la infancia. España: Innova

7. CRUZ HERNANDEZ, Hilda. - Escenario Educativo. Año 2011.

8. Arranz, M., \& García, C. (2011). Didáctica de la Educación Infantil. España: Paraninfo.

9. Blasco, J., \& Pérez, J. (2012). Metodología de investigación en las ciencias de la actividad física y el deporte. España: ECU.

10.Gallardo, D. (2017). Pedagogía a Distancia. Obtenido de http://pedagogia-adistancia.blogspot.com/2011/03/glosario-de-teoria-de-la-educacion.html

11. García, J. (2014). Ambietene con recursos tecnológicos. Costa Rica: EUNED.

12. Heredia, Y., \& Sánchez, A. (2013). Teorías del aprendizaje en el contexto educativo. México: Editorial digital tecnológico de Monterrey.

13. Hernández Sampieri, R., Fernández Collado , C., \& Baptista , L. (2014). Metodología de la investigación. México: Editorial Mc - Graw - Hill Interamericana. Sexta edición.

14. Lara, J. (2015). Las TIC en el escenario educativo. Obtenido de http://www.unitecnologica.edu.co/educacionadistancia/newletter/2015/boletin0 14/noti_apliaciones/005/index.html

15. Maya, E., Aldana Zavala, J., \& Isea Argüelles, J. (2019). Liderazgo Directivo y Educación de Calidad. CIENCIAMATRIA, 5(9), 114 - 129. https://doi.org/10.35381/cm.v5i9.102

16. Morgado, C., Aldana Zavala, J., \& Isea Argüelles, J. (2019). Gerencia transformacional desde el accionar docente directivo para el abordaje del Contexto Escolar. CIENCIAMATRIA, 5(9), 48-72. https://doi.org/10.35381/cm.v5i9.99 
17.Peche Cruz, H., \& Giraldo Supo, V. (2019). El Aprendizaje Flip Learning centrado en el estudiante como generador de calidad educativa. Revista Arbitrada Interdisciplinaria Koinonía, 4(8), 427-450. doi: HYPERLINK "http://dx.doi.org/10.35381/r.k.v4i8.293" http://dx.doi.org/10.35381/r.k.v4i8.293

18. Ramírez, Á. (2013). Pedagogía para aprendizajes productivos. Colombia: ECOE.

\section{REFERENCES CONSULTED}

1. Aldana, J., Salón, M., \& Guzmán, N. (2019). Systemic leadership in university teaching management skills. SCIENCE, 5 (8), 50-74. Recovered from http://cienciamatriarevista.org.ve/index.php/cm/article/view/87

2. ARRANZ, M., \& GARCIA, C. (2011). Teaching children's education. Spain: Paraninfo.

3. BIXIO, Cecilia. - New-Educational-Scenarios. Year 2010.

4. BLASCO, J., \& PEREZ, J. (2012). Research methodology in the sciences of physical activity and sports. Spain: ECU

5. CAUSIL GONZÁLEZ, Gustavo Adolfo. - Educational Environment. Year 2013.

6. CORDIVA, D. (2011). Cognitive, sensory, motor and psychomotor development in childhood. Spain: Innova

7. CRUZ HERNANDEZ, Hilda. - Educational stage. Year 2011.

8. Arranz, M., \& García, C. (2011). Teaching children's education. Spain: Paraninfo.

9. Blasco, J., \& Pérez, J. (2012). Research methodology in the sciences of physical activity and sports. Spain: ECU.

10.Gallardo, D. (2017). Distance Pedagogy Retrieved from http://pedagogia-adistancia.blogspot.com/2011/03/glosario-de-teoria-de-la-educacion.html

11.García, J. (2014). Ambietene with technological resources. Costa Rica: EUNED.

12. Heredia, Y., \& Sánchez, A. (2013). Learning theories in the educational context. Mexico: Monterrey digital technology publisher. 
13. Hernández Sampieri, R., Fernández Collado, C., \& Baptista, L. (2014). Investigation methodology. Mexico: Editorial Mc - Graw - Interamerican Hill. Sixth edition.

14.Lara, J. (2015). ICT in the educational scenario. Retrieved from http://www.unitecnologica.edu.co/educacionadistancia/newletter/2015/boletin0 14/noti_apliaciones/005/index.html

15.Maya, E., Aldana Zavala, J., \& Isea Argüelles, J. (2019). Leadership Leadership and Quality Education. CIENCIAMATRIA, 5 (9), 114-129. Https://doi.org/10.35381/cm.v5i9.102

16. Morgado, C., Aldana Zavala, J., \& Isea Argüelles, J. (2019). Transformational management from the directive teaching actions to address the School Context. SCIENCE, 5 (9), 48-72. https://doi.org/10.35381/cm.v5i9.99

17.Peche Cruz, H., \& Giraldo Supo, V. (2019). The student-centered Flip Learning Learning as a generator of educational quality. Interdisciplinary Arbitrated Review Koinonía, 4 (8), 427-450. doi: http: //dx.doi.org/10.35381/r.k.v4i8.293

18. Ramírez, Á. (2013). Pedagogy for productive learning. Colombia: ECOE.

(C2020 por los autores. Este artículo es de acceso abierto y distribuido según los términos y condiciones de la licencia Creative Commons Atribución-NoComercial-Compartirlgual 4.0 Internacional (CC BY-NC-SA 4.0) (https://creativecommons.org/licenses/by-nc-sa/4.0/). 